\title{
Enhancing nanocrystalline material yield strength during ARB process by using Solute atoms as reinforcement
}

\author{
P.B. Sob \\ Department of Mechanical Engineering, Faculty of Engineering and Technology, Vaal University of Technology. \\ Vanderbijlpark, South Africa
}

\begin{abstract}
In the current study solute atoms are used as reinforcement during ARB process. During the process of ARB dislocation line entanglement usually occurs in the grains, causing the formation of a jog which opposes dislocation motion during material deformation. These entanglements and jogs act as pinning points in the material during ARB process. The mobility of materials dislocation are more complex when the material are being deformed at elevated temperature as grain growth also takes place that negatively impacts the yield stress. To prevent grain migration and dislocation and even transfer of heat from one grain to other solute atoms are used as reinforcement during ARB process. Little studies have been done on thermal stability by studying the pinning points in the material and enhancing the material property to be more stable with high control dislocation process during grain refinement. In the current study focus is giving in material stability during grain refinement at elevated temperature by studying the dislocation of grain boundaries mobility. The following facts are revealed in modeling. It was shown that the yield stress increase to an ultimate yield stress which was accompanied by decrease in yield stress as hardness during particle cutting and hardness for particle bowing decreases. It was also shown that the change in yield stress and shear stress increase with time during ARB process. It was shown that the creation of second phase might also be created by mechanical or thermal treatments process during ARB. It is observed that the particles that compose the second phase cause pinning points.
\end{abstract}

Keywords: Yield stress, hardness, ARB, temperature and size

\section{Introduction}

Severe plastic deformation induced grain growth has been widely reported in various plastic deformation techniques including nano-indentation, high pressure torsion, uniaxial tension and uniaxial compression [1-5]. The grain growth changes the structures of nanocrystalline materials and therefore affects their mechanical properties [1-7]. Molecular dynamics simulations and theoretical analysis have been carried out to understand the mechanisms of deformation inducing grain growth, and contradictory results have been reported [1-9]. Grain rotation and coalescence process takes place when grains rotate and join as a single grain. It brings about a change in the size of a grain at any instant when the misorientation angle between some adjacent grains becomes zero [8-13]. The time evolution process shows grain rotation of grain A towards grain $B$ and coalescence with grain $B$ resulting in elongated grain A-B as shown in Figure $1(a-b)$ [9]. In the initial configuration the two grains are disoriented by approximately 180 [9]. During the initial deformation grain $\mathrm{A}, \mathrm{B}$ and $\mathrm{C}$ shrink due to GB migration, while grain $\mathrm{B}$ has already undergone some significant rotation towards the orientation of grain A [9]. It is further observed that grain B, has actually grown again due to continuous rotation [9]. It is also important to know that the inclination of GB migration between grains $\mathrm{A}$ and $\mathrm{B}$ has changed during this process. Finally the coalescence of grains A and B is observed in Figure 1(b) where two grains now being closely aligned with low-angle GB between them was reduced to single dislocation [9].

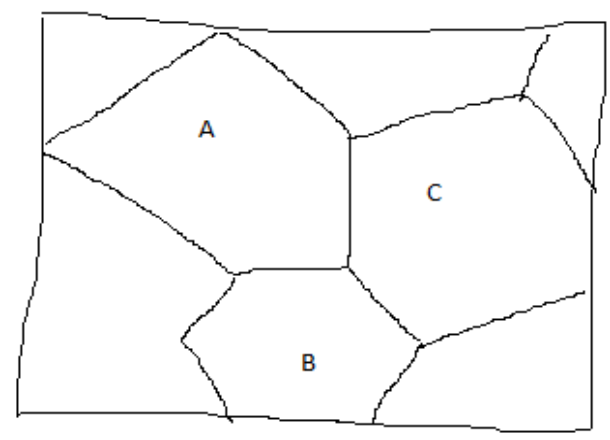


(a)

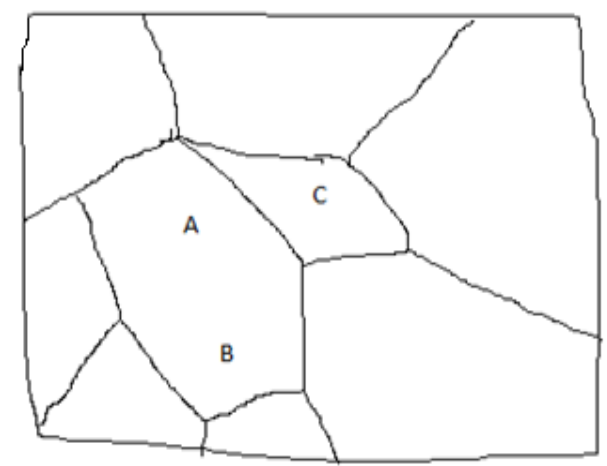

(b)

Figure.1. Grain Rotation Processes (Haslam et al. 2001:22).

Figure 2 (a) is a complementary view of grain rotationcoalescence mechanism [9]. The diagram in Figure 1 (b) revealed that rotation-coalescence may be seen as "unzipping" of the GB between the two grains with larger angle approximately 180 and high concentration of miscoordination in the GB between grain $\mathrm{A}$ and $\mathrm{B}$ [9]. Figure 1 (a-b) showing a very high concentration of miscoordination in the GB between grains $\mathrm{A}$ and $\mathrm{B}$ after a very short period. It is observed in Figure 2 (a) that the misorientation angle has reduced to approximately 16.50 with the creation of a dislocation structure of the GB. Three well separated GB dislocations can be observed in Figure 2 (a) and after a short period of time the misorientation angle decreased to about 90 and the dislocations left are shown in Figure 2 (b). The change in inclination of the GB between grains A and B observed in Figure 2 (c) and Figure 2 (d) coincides with this absorption. With the low remaining misorientation angle of about 40 after a short period of time as shown in Figure 2 (d) only the dislocation at the centre of the GB remains.

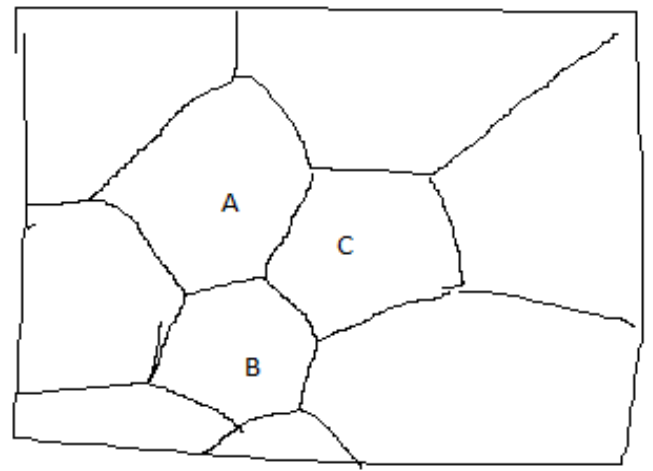

(a)

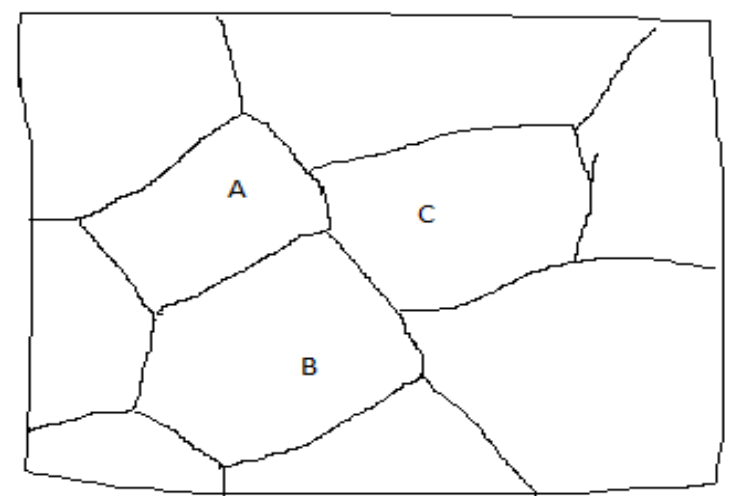

(b)

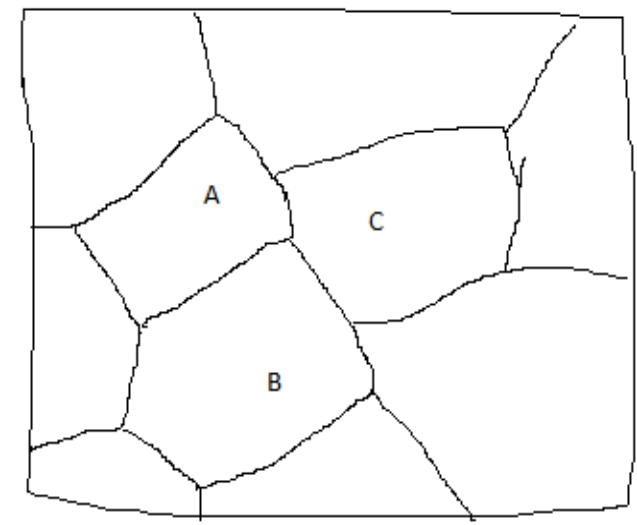

(c)

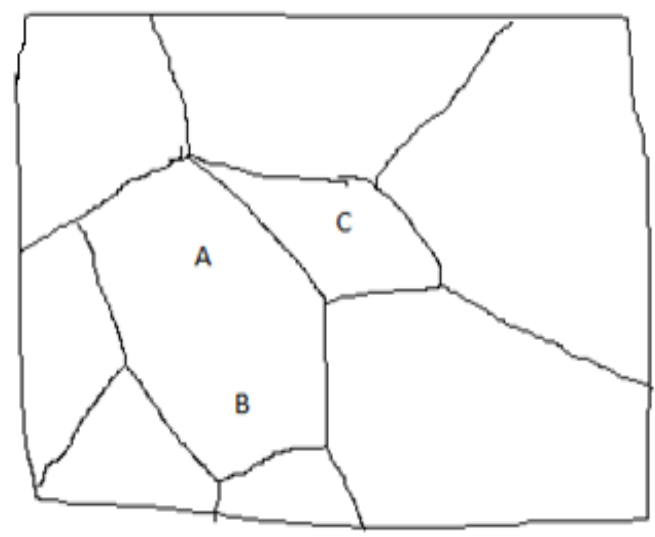

(d)

Figure.2. Grain Rotation Processes and Coalescence [9].

The force law governing GB migration and GR is given by Newton's law of motion coupling the acceleration of the atoms to the force acting on them [9]. A constant driving force for migration, $P$ results in a constant drift velocity 
given by $v=m p_{\text {[9] where }} m_{\text {is the GB mobility, which }}$ is a parameter such as the GB energy and it depends on the GB misorientation and the crystallographic orientation of the GB plane [9]. From the viscous force law $v=m p$ the mobility is independent of both the driving force and the mechanism of GB migration (Haslam et al., 2001:22). Since the applied force is at an angle, the mobility can be expressed with a force law which is similar to angular velocity of a rotating grain, $\omega_{\text {with respect to an axis }}$ through its centre of mass $\omega=M \tau_{\text {(Haslam et al., }}$ 2001:22) where $\mathbf{M}$ is the rotational mobility of the grain subjected to a torque $\tau$.

It is reported that some grains experience a net torque for rotation, provided that the total GB energy across all the surface of the neighbouring grains decreases as a result of the rotation (Haslam et al., 2001:22). The rotational mobility of a non-spherical grain embedded in a more or less rigid matrix of all the surrounding grains is strongly influenced by its shape (Haslam et al., 2001:22). The rotation of such a grain necessitates a continuous accommodation process (Haslam et al., 2001:22). Two accommodation mechanisms based on GB diffusion or dislocation motion exist (Haslam et al., 2001:22). At high temperatures the diffusionaccommodation process is more acceptable assuming a hexagonal grain shape within a columnar microstructure and diffusion acts as the accommodation mechanism (Haslam et al., 2001:22). It must be noted that Plastic deformation usually occurs when large numbers of dislocations move and multiply during grain refinement process [1-7]. To enhance the mechanical property in a material (i.e. increase the yield and tensile strength), it is vital to introduce a mechanism which prohibits the mobility of these dislocations during grain refinement. This mechanism may be, (work hardening, grain size reduction, etc.) since they hinder dislocation motion and render the material yield stress and tensile strength stronger [1-6]. The stress required to cause dislocation motion is orders of magnitude lower than the theoretical stress required to shift an entire plane of atoms, so this mode of stress relief is energetically favorable during grain refinement process. Therefore material yield and tensile strength greatly depend on the ease with which dislocations mobility during grain refinement. Normally the pinning points, or locations in the crystal that oppose the motion of dislocations during grain refinement can be introduced into the lattice to reduce dislocation mobility, thereby increasing mechanical strength of the material during grain refinement [5]. Material dislocations process may be pinned due to stress field interactions in the material during deformation process with other dislocations and solute particles, creating physical barriers from second phase precipitates forming along grain boundaries during grain refinement process. Usually there are five main strengthening mechanisms for metals. Each of this strengthening mechanism prevents dislocation motion and propagation, or makes it energetically unfavorable for the dislocation to move during grain refinement process. For a material that has been strengthened, by some processing method, the amount of force required to start plastic deformation is greater than it was for the original material.

Material strengthening is mainly caused by grain dislocations process. Dislocations interact with each other and create fields in the material during deformation. The interaction between the stress fields of dislocations during grain refinement might impede dislocation motion by repulsive or attractive interactions during deformation. During grain refinement process, if two dislocations cross during refinement, dislocation line entanglement usually occurs in the grains, causing the formation of a jog which opposes dislocation motion during material deformation. These entanglements and jogs act as pinning points in the material and this oppose dislocation motion. As both of these processes are more likely to occur when more dislocations are present, there is a correlation between dislocation density and yield strength. The mobility of materials dislocation is more complex when the material is being deformed at elevated temperature as grain growth also takes place that negatively impacts the yield stress and tensile strength of the material. Thermal stability in nanomaterials is a hot spot area in materials study due to the complexity of grain growth. Little studies have been done on thermal stability by studying the pinning points in the material and enhancing the material to be more stable with high control dislocation process during grain refinement. In the current study focus is giving in material stability during grain refinement at elevated temperature by studying the dislocation of grain boundaries mobility.

\section{Methodology}

Material hardenings are mainly caused by dislocations during grain refinement. The dislocations interact with each other and generate field stresses in the material during grain refinement. The field stress interaction caused by dislocation impedes dislocation motion due to their repulsive or attractive interactions during grain refinement. 
During this process, if two dislocations cross there is a possibility of dislocation line entanglement occurs which usually leads to the formation of a jog which opposes dislocation motion during grain refinement. These jogs and entanglements that are created normally act as pinning points, which oppose dislocation motion during grain refinement. Since these activities are more likely to occur only due to the present of more dislocations, therefore there is a clear correlation between dislocation density and material hardening (yield strength) and this relationship can be given as,

$\Delta \sigma=G b \rho 1^{1 / 2}$

where $\mathrm{G}$ is the shear modulus, $\mathrm{b}$ is the Burgers vector, and $\rho 1$ is the dislocation density. The dislocation density was computed using the formula $\rho 1=\frac{1}{D^{3}}=\frac{1}{r^{3}}$ where $D=r=$ the particle sizes. If the directions of all dislocation lines are assumed to run from/to the node, then the sum of the Burgers vectors of the dislocations is zero the assumption is very similar to Kirchhoff's law. During grain refinement process, increasing the dislocation density increases the yield strength. Therefore higher shear stress will be required to move the dislocations during grain refinement. This is an indication that the strength of a material without dislocations is usually high due to the fact that plastic deformation usually involves the breaking of many bonds. This requires high shear stress to move the dislocation during grain refinement. The shear stress to move dislocation is given as,

$\Delta \tau=G b \sqrt{c \varepsilon^{3 / 2}}$

Where $\mathrm{c}$ is the solute concentration, $\varepsilon$ is the strain in the material caused by solute. By increasing the concentration of the solute atoms leads to an increase in yield stress of nanomaterial during ARB as shown in Fig.3 (a-b).

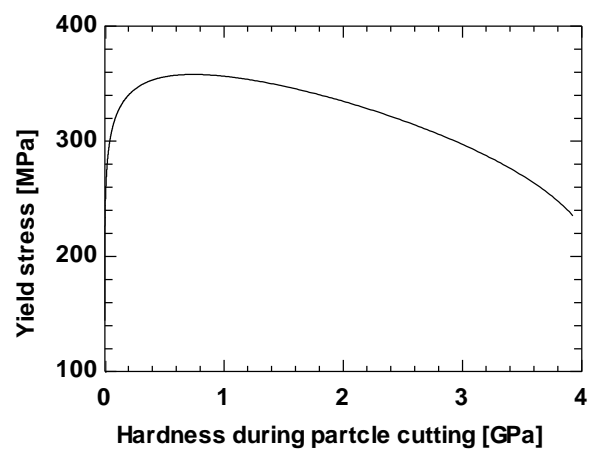

(a)

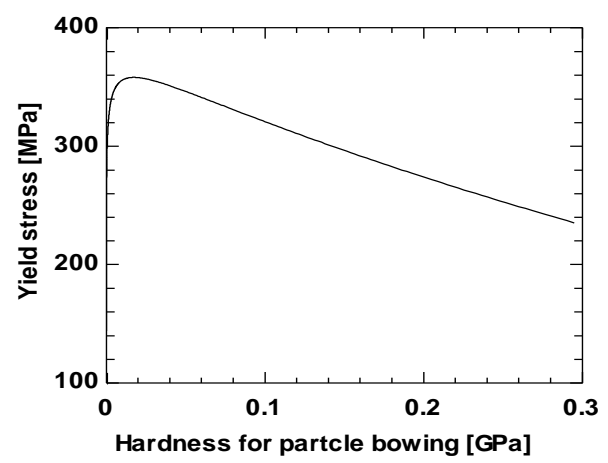

(b)

Figure.3: (a) Yield stress and hardness during particle cutting (b) Yield stress and hardness for particle bowing

It is shown in Fig.3 (a-b) that the yield stress increase to an ultimate yield stress which was accompanied by decrease in yield stress as hardness during particle cutting and hardness for particle bowing decreases. This is due to the fact that there is a limit to the amount of solute atoms that can be added to the material during ARB cycles. If excess solute atoms are added to material during ARB a second phase is created which lower the material yield stress. Normally the solid solution stress and strengthening property depends on the following factors (a) the concentration of the material solute atoms, (b) the material shear modulus of solute atoms, (c) the size of solute atoms of the material, (d) the material valency of the solute atoms for specific material such as ionic materials, and (e) the symmetry of the solute stress field in the material during ARB. This is due to the fact that the magnitude of strengthening during deformation is higher for non-symmetric stress fields due to the fact that the solutes atoms can interact with both edge and screw dislocations during deformation. Therefore the change in yield stress, shear stress increases with time during ARB as shown in Fig. 4 (a-c). 


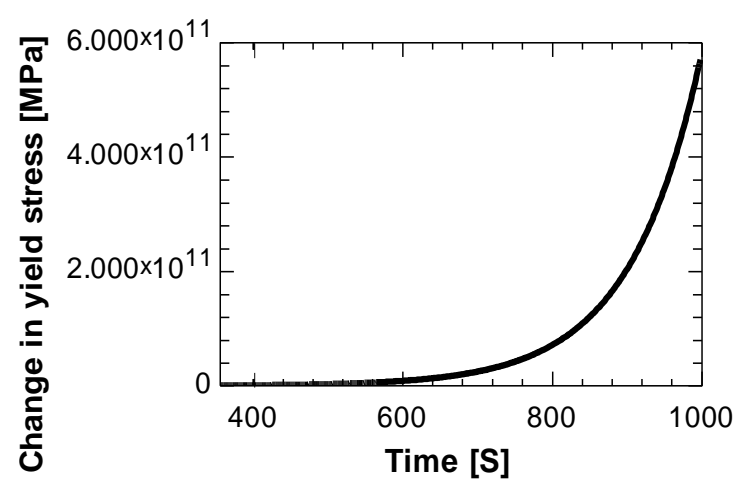

(a)

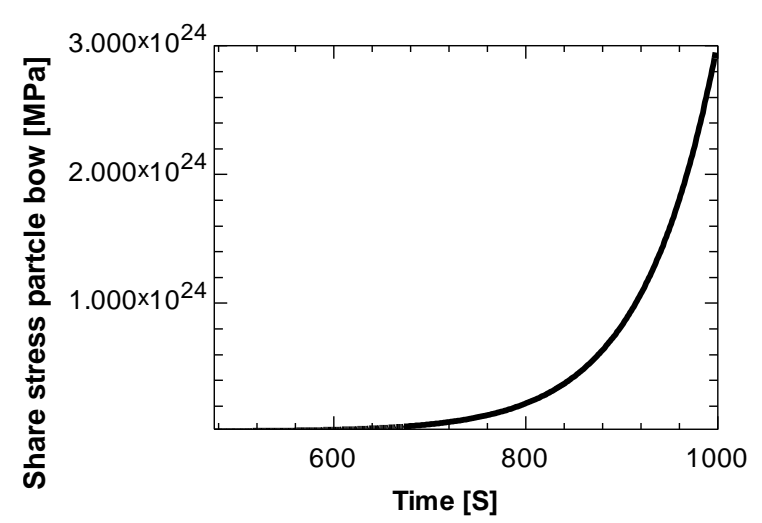

(b)

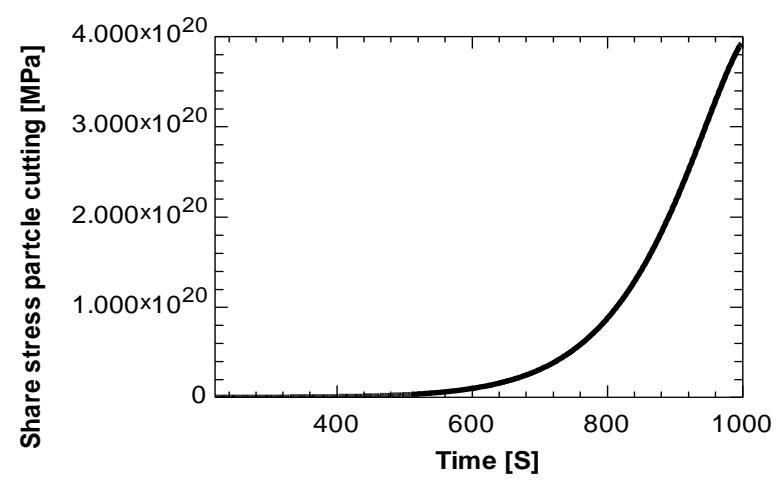

(c)

Figure.4: (a) Change in yield stress and time during ARB (b) Shear stress in particle bow and time during ARB (c) Shear stress in particle cutting and time during ARB.

The results in Fig.4 (a-c) revealed that the change in yield stress and shear stress increase with time during ARB process. This is due to the following reasons. During ARB cycles there are possibility of formation other phases such as second phase. The creation of second phase might also be created by mechanical or thermal treatments process during
ARB. It is observed that the particles that compose the second phase causes pinning points. The material dislocations interact with the pinning points leading to increase in shear stress during ARB as shown in Fig. 4 (ab). During this process, if the precipitate atoms are small, the dislocations might cut through atoms in the material creating new surfaces. Usually for larger precipitate atoms, looping or bowing of the dislocations might occur and this will leads to the dislocations motion getting longer during ARB. This will affect the material hardness as deformation takes place since temperature increases during ARB.

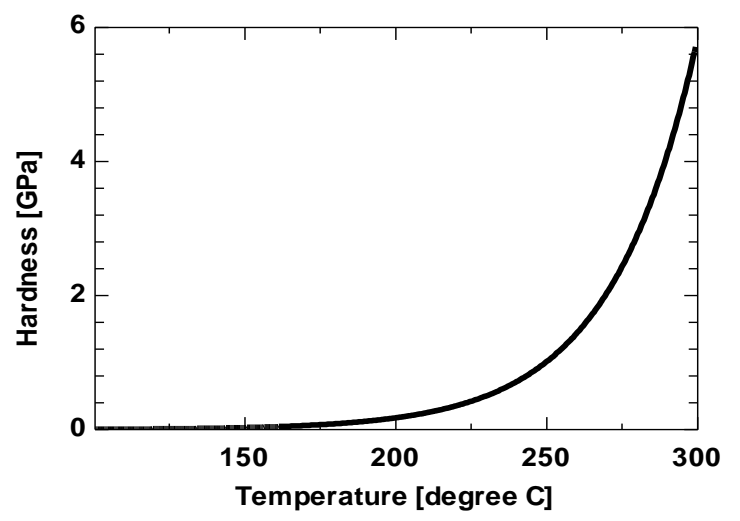

(a)

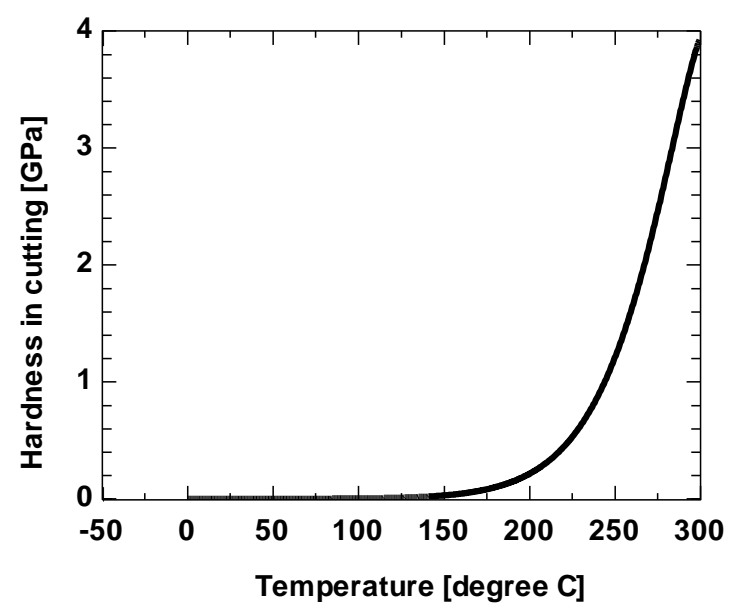

(b)

Figure.5: (a) Hardness and temperature during ARB (b) hardness in cutting and temperature

Normally during ARB process, material with bigger grain size have more dislocation to pile up leading to greater driving force for dislocations to move from one grain to another during ARB. In nanocrystalline material, grain size has a great influence on hardness. This is due to the fact that grains usually have varying crystallographic orientations 
International Journal of Engineering Research and Technology. ISSN 0974-3154, Volume 13, Number 9 (2020), pp. 2519-2525

(C) International Research Publication House. https://dx.doi.org/10.37624/IJERT/13.9.2020.2519-2525

and grain boundaries. During grain refinement slip motion will take place and the grain boundaries act as an impediment to dislocation motion. The material dislocation must change its direction of motion due to the differing orientation of grains during ARB and high stress is needed to move a dislocation. As this happens the temperature increases during ARB due to adiabatic warming. The material hardness increases though temperature increases as shown in Fig. 5 (a-b) mainly due to the solute atoms that prevent transfer of heat that will lead to grain growth during ARB.

\section{Conclusion}

The current study was aimed at studying the effect of thermal activities on yield stress using solute atoms as reinforcement during ARB process. The physical parameters that are involved in during grain refinement of nanomaterial using solute atoms were taking into consideration. The following facts were revealed property enhancements were observed at high temperature during ARB process. There were entanglements and jogs that act as pinning points in the material during ARB process. By studying the pinning points in the material, it was shown that control dislocation process during grain refinement. It was also shown that the yield stress increase to an ultimate yield stress which was accompanied by decrease in yield stress as hardness during particle cutting and hardness for particle bowing decreases. It was also shown by simulation that there is a change in yield stress and shear stress increase during $\mathrm{ARB}$ as deformation time increase.

\section{Acknowledgement}

This material is based on the work which is supported financially by the Vaal University of Technology (VUT).

Reference

1. Davidge, R.W., Mechanical Behavior of Ceramics, Cambridge Solid State Science Series, (1979)

2. ^ Lawn, B.R., Fracture of Brittle Solids, Cambridge Solid State Science Series, 2nd Edn. (1993)

3. $\wedge$ Green, D., An Introduction to the Mechanical Properties of Ceramics, Cambridge Solid State Science Series, Eds. Clarke, D.R., Suresh, S., Ward, I.M. (1998)
4. ^ $\underline{\text { Jump up to: }}:^{a} \underline{\underline{b}} \underline{\underline{c}}$ Callister, William Jr, Materials Science and Engineering, An Introduction. John Wiley \& Sons, NY, NY (1985)

5. ^ Kuhlmann-Wilsdorf, D., "Theory of Plastic Deformation," Materials Science and Engineering A, vol 113, pp 1-42, July 1989

6. ^ Jump up to: ${ }^{a} \underline{\underline{b}} \underline{\underline{c}} \underline{\underline{d}}$ Meyers, Chawla. Mechanical Behavior of Materials. Cambridge University Press. pg 420-425. 1999

7. ^ Krutyeva, M.; Wischnewski, A.; Monkenbusch, M.; Willner, L.; Maiz, J.; Mijangos, C.; Arbe, A.; Colmenero, J.; Radulescu, A.; Holderer, O.; Ohl, M.; Richter, D. (2013). "Effect of Nanoconfinement on Polymer Dynamics: Surface Layers and Interphases". Physical Review Letters. 110 (10): 108303. doi:10.1103/PhysRevLett.110.108303. hdl :10261/102483. PMID 23521308.

8. ^ Ligon-Auer, Samuel Clark; Schwentenwein, Martin; Gorsche, Christian; Stampfl, Jürgen; Liska, Robert (2016). "Toughening of photocurable polymer networks: A review". Polymer Chemistry. 7 (2): 257286. doi:10.1039/C5PY01631B.

9. ^ Zhang, Min; Askar, Shadid; Torkelson, John M.; Brinson, L. Catherine (2017). "Stiffness Gradients in Glassy Polymer Model Nanocomposites: Comparisons of Quantitative Characterization by Fluorescence Spectroscopy and Atomic Force Microscopy". Macromolecules. 50 (14): $\quad 5447-$ 5458. doi:10.1021/acs.macromol.7b00917.

10. ^Arkles, Barry; Crosby, Jane (1989). "Polysiloxane-Thermoplastic Interpenetrating Polymer Networks". Silicon-Based Polymer Science. Advances in Chemistry. 224. pp. 181199. doi:10.1021/ba-1990-0224.ch010. ISBN $\underline{\text { O- }}$ 8412-1546-4.

11. ^ Singh, Naveen K.; Lesser, Alan J. (2010). "Mechanical and thermo-mechanical studies of double networks based on thermoplastic elastomers". Journal of Polymer Science Part B: Polymer $\quad$ Physics. 48 (7): 778789. doi: $10.1002 /$ polb.21943.

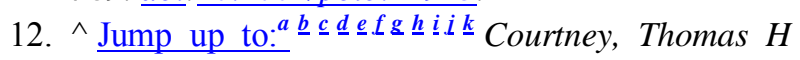
(1990). Mechanical Behavior of Materials (2nd ed.). Long Grove, IL: Waveland Press Inc.

13. ^ Jump up to: ${ }^{a}$ Buehler, Markus J. the dynamical complexity of work - hardening: a large-scale molecular dynamics simulation. Acta Mech Sinica. pg 103-111. 2005 
International Journal of Engineering Research and Technology. ISSN 0974-3154, Volume 13, Number 9 (2020), pp. 2519-2525

(C) International Research Publication House. https://dx.doi.org/10.37624/IJERT/13.9.2020.2519-2525

14. ^ Abraham, F. "Simulating materials failure by using up to one billion atoms and the world's fastest computer: Work-hardening." Proc Natl Acad Sci. pg 5783-5787. 2002

\section{AUTHORS' BACKGROUND}

\begin{tabular}{|l|l|l|l|l|}
\hline \multicolumn{1}{|c|}{ Your Name } & Position* & Email & Research Field & Personal website \\
\hline P.B SOB & Lecture & $\begin{array}{c}\text { Baonhe_sob@rocket } \\
\text { mail.com }\end{array}$ & $\begin{array}{l}\text { Nanomaterials, } \\
\text { wettability and solid } \\
\text { mechanic } \quad\end{array}$ & \\
\hline & & & & \\
\hline & & & & \\
\hline & & & & \\
\hline
\end{tabular}

